\title{
HIPERTENSÃO RESISTENTE: ANÁLISE DOS ESTUDOS PATHWAY 2 E 3
}

\author{
RESISTANT HYPERTENSION: ANALYSIS OF PATHWAY 2 AND 3 STUDIES
}

\author{
Fernando Focaccia Póvoa', Dilma de Souza², Rui Póvoa ${ }^{3}$
}

\section{RESUMO}

A hipertensão arterial resistente (HAR) é definida quando, apesar do tratamento com pelo menos três medicações anti-hipertensivas (incluindo um diurético) de diferentes classes a meta pressórica não é alcançada. Nesta sequência de fármacos, por muitos anos se utilizou empiricamente ou baseado em pequenos estudos, a espironolactona. Os estudos Pathway 2 e 3 vieram para corroborar a importância deste quarto fármaco, a espironolactona, como o mais eficaz em termos de potencia anti-hipertensiva, como também explicar os aspectos fisiopatológicos que levam o hipertenso a ficar resistente. Nesta revisão e análise crítica dos fármacos anti-hipertensivos na HAR destacamos os principais mecanismos envolvidos no não controle da pressão e as estratégias para um melhor controle pressórico.

Descritores: Hipertensão Arterial Resistente; Anti-Hipertensivos; Espironolactona; Amilorida.

\section{ABSTRACT}

Resistant arterial hypertension (RAH) is defined when, despite treatment with at least three antihypertensive medications (including a diuretic) of different classes, the pressure target is not achieved. In this sequence of drugs, for many years it was used empirically or based on small studies, spironolactone. Pathway 2 and 3 studies have come to corroborate the importance of this fourth drug, spironolactone, as the most effective in terms of antihypertensive potency, as well explain the pathophysiological aspects that lead hypertensive patients to become resistant. In this review and critical analysis of antihypertensive drugs in hypertension, we highlight the main mechanisms involved in the lack of pressure control and the strategies for better pressure control.

Keywords: Resistant Arterial Hypertension; Antihypertensive Drugs; Spironolactone; Amiloride.

\section{INTRODUÇÃO}

Desde o final do século passado a hipertensão arterial resistente (HAR) vem sendo estudada pelas diferentes sociedades internacionais. Com o aumento da expectativa de vida a HAR vem se tornando um problema de saúde pública com alta morbimortalidade e assim, diversos estudos propuseram novos esquemas terapêuticos, além de medidas intervencionistas para o controle pressórico. ${ }^{1}$

A senescência, obesidade, diabetes mellitus, apneia do sono, hipertrofia ventricular esquerda e a ingesta excessiva de sódio contribuem exponencialmente o risco de desenvolver a HAR. ${ }^{2-5}$

A definição de HAR é quando, apesar do tratamento com pelo menos três medicações anti-hipertensivas (incluindo um diurético) de diferentes classes na combinação correta e nas doses alvo toleradas a meta pressórica não é alcançada. ${ }^{6}$

Pacientes cuja meta pressórica (medida da pressão arterial de consultório >140/90 mmHg) é alcançada com quatro ou mais medicamentos anti-hipertensivas são definidores de HAR controlada. ${ }^{7}$

\section{HAR COMO UM FATOR DE RISCO CARDIOVASCULAR ADICIONAL}

A prevalência da HAR é desconhecida e o entendimento advém de ensaios clínicos controlados. Os dados do National Health and Nutrition Examination Survey de 2003-2008 demonstram que $8,9 \%$ dos norte-americanos preencheram os critérios para HAR, representando $12,8 \%$ dos pacientes tratados com anti-hipertensivos. De todos os adultos tratados com drogas anti-hipertensivas, $72,4 \%$ dos pacientes não estavam com a hipertensão controlada tomando até três fármacos. De forma semelhante a hipertensão não resistente, a HAR é também multifatorial, com mesmas características genéticas e ambientais, no entanto o sal se torna um importante coadjuvante na sua fisiopatologia. ${ }^{8}$

\footnotetext{
1. Emergências Clínicas da Faculdade de Ciências Médicas de Santos- Universidade Lusíada. SP, Brasil

2. Disciplina de Cardiologia da Faculdade de Medicina da Universidade Federal do Pará, PA, Brasil

3. Disciplina de Cardiologia da Universidade Federal de São Paulo, SP, Brasil.

Correspondência:

http://dx.doi.org/10.47870/1519-7522/2021280114-19
} 
O estudo ELSA, Estudo Longitudinal Brasileiro de Saúde do Adulto, por meio de uma coorte com 15.105 indivíduos entre 35 e 74 anos os autores identificaram 4.116 pacientes em tratamento para hipertensão, sendo que $11 \%$ eram portadores de HAR. ${ }^{9}$

Diversos estudos observacionais mostraram que pacientes com HAR apresentam desfechos desfavoráveis em comparação aos pacientes sem HAR. Em um estudo prospectivo com mais de 200.000 pacientes com hipertensão incidente, os pacientes com HAR foram $47 \%$ mais propensos em sofrer eventos combinados de morte, infarto do miocárdio, insuficiência cardíaca, acidente vascular encefálico ou doença renal crônica ao longo de 3,8 anos de seguimento. ${ }^{10}$

Estudos prospectivos com uso da monitorização ambulatorial da pressão arterial (MAPA) sugerem um risco aumentado de duas vezes para desenvolver eventos cardiovasculares em pacientes com HAR em comparação com os pacientes hipertensos que respondem adequadamente ao tratamento anti-hipertensivo. ${ }^{11}$

Afastar as principais causa de pseudorresistência se tornam primordial na abordagem do paciente com HAR. A má técnica da aferição da pressão arterial (especialmente o tamanho do manguito para pacientes obesos), má aderência medicamentosa e não medicamentosa e a associação inadequada de fármacos, ou doses inadequadas são pontos que devem ser exaustivamente pesquisados frente ao paciente cuja cifras pressóricas não são alcançadas com o tratamento clínico otimizado. A escolha do medicamento é de extrema importância, principalmente a sua dosagem, bem como o uso de duas drogas sinérgicas que reduzem morbimortalidade cardiovascular. ${ }^{6,12,13}$

A baixa adesão medicamentosa não pode ser atribuível simplesmente ao esquecimento de ingerir as medicações, mas também ao alto custo, efeitos colaterais e baixa escolaridade. Reduzir o número de tomadas diárias, educar em relação os efeitos colaterais e a conscientização do uso contínuo das medicações anti-hipertensivas, em conjunto com medidas não farmacológicas, são etapas importantes para fortalecer a adesão. Somente após esses ajustes e caso a pressão de consultório permanecer elevada, os pacientes podem ser diagnosticados como hipertensos resistentes. ${ }^{14}$

\section{DIAGNÓSTICO DA HAR}

Embora a definição de HAR seja baseada nas aferições da pressão arterial no consultório, a MAPA é crucial para uma melhor avaliação e seguimento dos pacientes, por causa do efeito do avental branco e a avaliação do comportamento da pressão no período noturno. ${ }^{6,15,16}$

Desta forma, a MAPA distingue o verdadeiro hipertenso resistente (pressão arterial de consultório $\geq 140 / 90 \mathrm{mmHg}$, $\geq 135 / 85 \mathrm{mmHg}$ durante o dia ou $\geq 120 \times 70$ durante o sono) e a hipertensão resistente do jaleco branco (pressão arterial do consultório $\geq 140 / 90 \mathrm{mmHg}$ e tanto a pressão diurna e a noturna com $<135 / 85$ mmHg e $<120 \times 70$ mmHg, respectivamente). A medida residencial da pressão da pressão arterial, com aparelho calibrado e validado, pode ser uma alternativa a MAPA, embora pareça superestimar a pressão arterial e não detectar a resistência noturna. ${ }^{11}$
Todos os pacientes devem realizar exames laboratoriais avaliando o perfil metabólico devido à alta prevalência de doenças preexistentes como diabetes e dislipidemia. A função renal (creatinina sérica, microalbuminúria e proteinúria) devido a associação com doença renal crônica apresenta uma importância prognóstica e evidências atuais demonstram um risco aumentado de eventos adversos cardiovasculares e renais em pacientes com HAR em comparação aos hipertensos controlados. O risco de o paciente evoluir para o último estágio da doença renal crônica é de 25 \% em pacientes com HAR. 6,16,17

O eletrocardiograma deve ser realizado para identificar hipertrofia ventricular esquerda e fornece importantes marcadores prognóstico com alteração da repolarização ventricular, padrão strain e critérios de hipertrofia ventricular esquerda. ${ }^{18}$

As causas secundárias de hipertensão podem ser surpreendentemente comuns em pacientes com HAR e o hiperaldosteronismo é uma das mais frequentes, culminando em $20 \%$ dos pacientes resistentes. ${ }^{19}$

\section{SEGUIMENTO DO PACIENTE COM HAR}

Atualmente muitos autores referem uma importante associação entre hiperaldosteronismo e a hipertensão não controlada, além de ser um marcador de gravidade na doença cardiovascular. Comumente definido como aldosteronismo primário, a maioria dos casos não é representada pelo clássico adenoma adrenal. Caso contrário, a hiperplasia unilateral da adrenal pode ser tratada cirurgicamente, mas o bloqueio mineralocorticoide é uma opção. ${ }^{19-21}$

A apneia obstrutiva do sono e a hipertensão também estão intimamente relacionados. Os principais sintomas são sonolência diurna e fadiga, associados a distúrbios respiratórios obstrutivos (apneia ou hipopneias). Em algumas séries a prevalência da apneia obstrutiva atinge $65-80 \%$ em pacientes com hipertensão resistente. Diversos mecanismos são propostos para explicar como apneia obstrutiva do sono causa resistência ao tratamento, incluindo a retenção de líquidos em torno do pescoço, estresse oxidativo e disfunção endotelial. O tratamento é ainda controverso, contudo, é bem estabelecido que pacientes com apneia obstrutiva do sono grave, cifras pressóricas elevadas, níveis elevados de hipopneia e apneia se beneficiam com o uso do CPAP (Contiunous Positive Airway Pressure). ${ }^{4,22,23}$

Apesar de diversas opções terapêuticas, é de importância alcançar a adesão medicamentosa e não medicamentosa ao longo da vida do paciente. A abordagem multidisciplinar como mudança no estilo de vida e o tratamento farmacológico intensivo se tornam necessário.

A mudança no estilo de vida é o primeiro passo no tratamento de qualquer paciente hipertenso e a redução na ingesta salina, aumento da atividade física e redução do peso são pilares primordiais. A restrição salina tem eficácia inquestionável e recentemente foi demonstrado em um estudo randomizado que o baixo teor de sal reduziu a pressão arterial sistólica e diastólica na MAPA em $20 \mathrm{mmHg}$ e $10 \mathrm{mmHg}$, respectivamente. Todos os esforços para perda de peso, com exercícios físicos, devem ser encorajados nos pacientes hipertensos resistentes. ${ }^{6,12-24}$

Depois de considerar as modificações no estilo de vida, é importante focar no esquema terapêutico apropriado. 
Segundo o fluxograma do tratamento os três principais fármacos apropriados são um inibidor do sistema renina angiotensina aldosterona (iSRAA) mais um antagonista dos canais de cálcio (ACC) mais um diurético tiazida (DIUt). Pacientes com HAR frequentemente apresentam retenção de volume subclínico e a terapia diurética é essencial para o controle da pressão arterial. Diuréticos tiazídicos são eficazes na maioria dos pacientes com HAR, e a clortalidona, por sua ação mais longa, é preferida quando comparada com a hidroclorotiazida. Os diuréticos de alça são preferíveis em pacientes com doença renal crônico quando o clearance creatinina for < $30 \mathrm{ml} . \mathrm{min}^{-1}$ por $1,73 \mathrm{~m}^{2}$ Neste caso a furosemida deve ser prescrita idealmente com uma segunda dose após 6-8h devido a sua curta ação. ${ }^{6,19}$

\section{ESTUDO PATHWAY-2}

A HAR é um estado heterogêneo principalmente causado pela retenção de sódio e o estudo PATHWAY-2 (The Prevention And Treatment of Hypertension With Algorithm based therap $Y$ ) escolheu a espironolactona (bloqueador dos receptores de mineralocorticóides), como o quarto fármaco. ${ }^{25}$

Foi um estudo duplo cego, placebo controlado e cruzado ("crossover"), fase 4 com 12 meses de follow-up cujos pacientes foram selecionados de 12 centros de atenção secundária e dois centros de atenção primária do Reino Unido. O ensaio clínico envolveu pacientes com idade entre 18-79 anos com pressão arterial sistólica de consultório $\geq 140 \mathrm{mmHg}$ (ou $\geq 135 \mathrm{mmHg}$ para pacientes diabéticos) e pressão arterial sistólica pela medida residencial da pressão arterial (18 leituras do aparelho ao longo de 4 dias) $\geq 130 \mathrm{mmHg}$. Todos os pacientes estavam em tratamento clínico por pelo menos três meses com as doses máximas toleradas das três drogas (iSRAA + DIUt + ACC).

A randomização do estudo foi para o uso de espironolactona (25-50 mg), bisoprolol (5-10 mg), doxazosina (4-8 mg) ou placebo durante as 12 semanas sendo que a dose dos fármacos foi duplicada após 6 semanas.

Como a análise foi intenção de tratar, foi avaliada a diferença entra a pressão arterial sistólica fora do consultório (avaliação residencial) entre a espironolactona e os outros dois fármacos.

Foram incluídos 335 pacientes e após a exclusão de 21, 285 pacientes receberam espironolactona, 282 doxazosina, 285 bisoprolol e 274 placebo. Do total, 230 pacientes completaram todo o ciclo do tratamento. A redução média na pressão arterial sistólica no grupo espironolactona foi de $8,7 \mathrm{mmHg}$, superior ao placebo (IC $95 \%$ - 9,72 a -7,69; p <0,0001) e superior a média dos outros dois fármacos (doxazosina e bisoprolol; -4,26 [IC95\% -5,13 a -3,38]; p <0,0001), além da superioridade quando comparados separadamente. A comparação com a doxazosina, a redução foi $-4,03 \mathrm{mmHg}$ [IC95\% -5,04 a -3,02]; $p<0,0001$ ) versus o bisoprolol de $-4,48 \mathrm{mmHg}$ (IC 95\%, -5,50 a-3,46; $\mathrm{p}<0,0001$ ). (Figura 1)

A proporção de pacientes nos quais a espironolactona foi a melhor droga para redução das cifras pressóricas foi mais evidente nas análises planejadas por renina plasmática a cada droga e a probabilidade de que diferentes drogas seriam melhores em diferentes pontos da curva da renina plasmática.

Houve uma relação entre a renina plasmática e a queda pressórica ao tratamento ativo corrigido para o efeito placebo. Foi uma relação inversa entre a queda da pressão arterial sistólica domiciliar com a espironolactona e a quantidade de renina plasmática, não observado com o bisoprolol ou a doxazosina. (Figura 2)

A tolerabilidade do uso da espironolactona foi alta, apenas seis pacientes apresentaram potássio sérico acima de 6,0 mmol/l em uma ocasião.

O estudo PATHWAY-2 foi o primeiro ensaio clínico randomizado que comparou a espironolactona com as demais drogas que reduzem a pressão arterial em uma população bem definida de hipertensos resistentes. O estudo sugere que há uma predominância subjacente da causa fisiopatológica da HAR e a retenção de sódio, apesar do diurético pré-estabelecido na terapia existente, e é a principal explicação defendida pelos autores.

A espironolactona apresentou uma resposta inversamente proporcional com a renina plasmática e foi efetiva em níveis

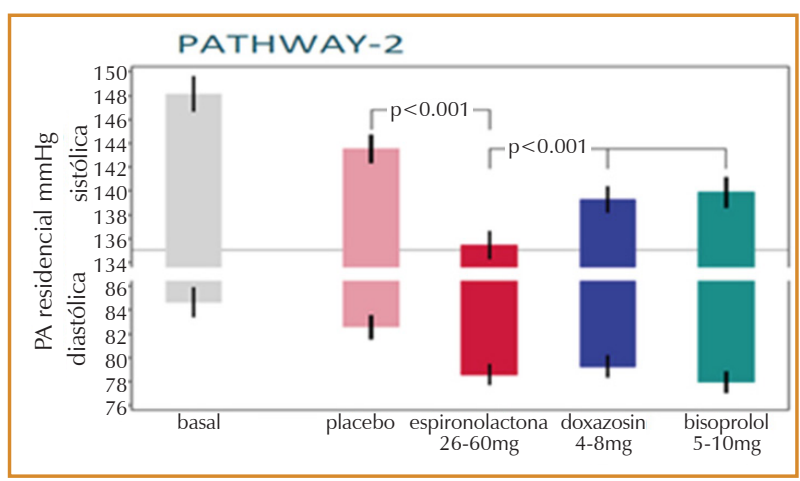

Figura 1. Pressão arterial residencial sistólica e diastólica na hipertensão resistente comparando a espironolactona com doxazosina e bisoprolol. Modificado da referência 25.

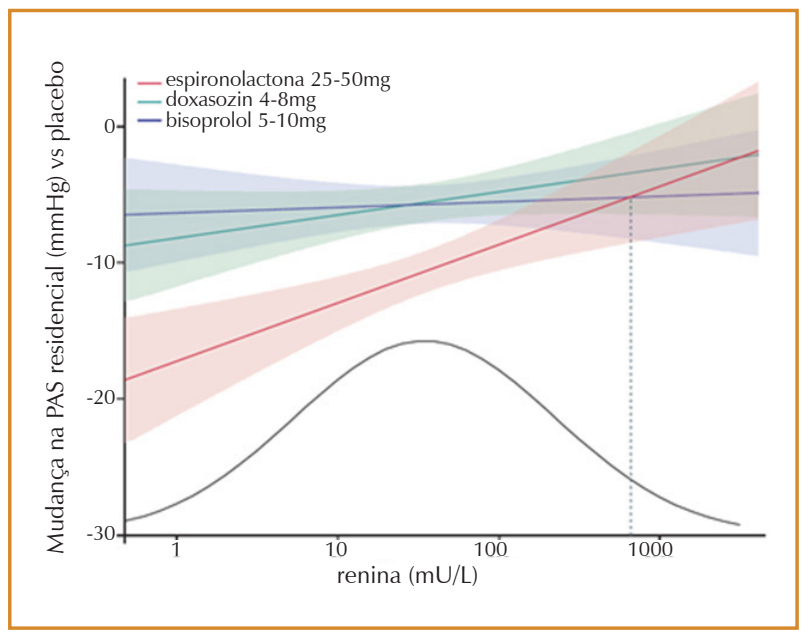

Figura 2. A distribuição da curva é ajustada à renina da linha de base observada no estudo. A linha tracejada vertical mostra a queda da pressão arterial com o bisoprolol apenas nos $3 \%$ da distribuição da renina. Legenda: PAS: Pressão arterial sistólica. Modificado da referência 25. 
de renina baixo, além de ser a droga mais eficaz em toda concentração de renina plasmática e alcançou a pressão arterial em relação ao bisoprolol ou doxazosina em aproximadamente $60 \%$ dentro de 3 meses após o início do tratamento. O bisoprolol e a doxazosina foram mais eficazes do que o placebo na redução da pressão arterial, mas significantemente menos eficaz do que a espironolactona, estabelecendo pela primeira vez uma hierarquia clara, como a quarta droga anti-hipertensiva, para o tratamento da HAR.

Importante ressaltar que o estudo utilizou da medida na residência, minimizando o efeito placebo, bastante evidente no estudo, e assim eliminou pacientes com o efeito do avental branco.

Estudos prévios ao PATHWAY-2 utilizavam de rotina observações na prática clínica e em pequenos relatos de caso. ${ }^{26}$

Contudo os resultados do PATHWAY-2 foram impactantes para consolidar a quarta droga para tratamento da HAR e provocando mudanças em diretrizes internacionais como a última diretriz europeia de hipertensão (Figura 3) ("2018 ESC/ ESH Guidelines for the management of arterial hypertension") seguindo a seguinte orientação:

"O início do tratamento na maioria dos pacientes deve ser feito com combinação de dois fármacos em uma única pílula, para melhorar a eficiência, velocidade e o controle da pressão arterial".

"As combinações de dois medicamentos preferidos são um inibidor do SRAA com um ACC ou um diurético, caso não haja uma indicação específica para o uso de um betabloqueador (angina, infarto ou insuficiência cardíaca)".

"O uso de uma única pílula contendo um inibidor do SRAA com ACC mais um diurético se a pressão não foi controlada com dois fármacos".

"A adição da espironolactona para o tratamento da HAR a menos que haja uma contraindicação".

\section{ESTUDO PATHWAY-3}

A depleção de potássio com o uso dos diuréticos tiazídicos está associado a hiperglicemia e o risco de desenvolver diabetes tipo 2. O estudo PATHWAY-3 avaliou se a adição ou substituição de um diurético poupador de potássio, a amilorida, no tratamento com um diurético tiazídico pode prevenir a intolerância a glicose e melhorar o controle pressórico.

O risco de desenvolver diabetes tipo 2 parece estar ligado a depleção de potássio e pode ser evitada pelo uso de diuréticos poupadores de potássio, que são convencionalmente consideradas a classe mais fraca de diuréticos pois a maioria do sódio filtrado é reabsorvido a montante de seu local de ação do néfron. No entanto, os diuréticos poupadores de potássio agem em um local comum na retenção de sódio, e pode ser essencial na prevenção de respostas compensatórias à ação mais proximal dos diuréticos tiazídicos e de alça. ${ }^{27-30}$

Assim, surgiu a hipótese que uma dose adequada de diurético poupador de potássio poderia ter efeitos opostos aos de um diurético tiazídico sobre o potássio e a glicose, mas teria efeitos semelhantes ou adicionais na pressão sanguínea quando os dois foram comparados ou combinados.

O estudo PATHWAY-3 com duração de 24 semanas, reuniu 11 centros de atenção secundária e dois centros de atenção primária no Reino Unido. ${ }^{31}$ Foi um estudo randomizado, duplo-cego e grupo paralelo (estudo clínico onde dois grupos de tratamentos são dados de modo que um grupo receba apenas uma droga enquanto outro grupo receba a outra medicação). Os critérios de inclusão foram: idade entre 18-20 anos, pressão arterial sistólica no consultório $\geq 140$ $\mathrm{mmHg}$, pressão arterial residencial $\geq 130 \mathrm{mmHg}$, indicação para uso de diurético como pressão arterial sistólica elevada apesar do tratamento com iSRAA, betabloqueador, ACC ou inibidor direto da renina e pelo menos, um componente de

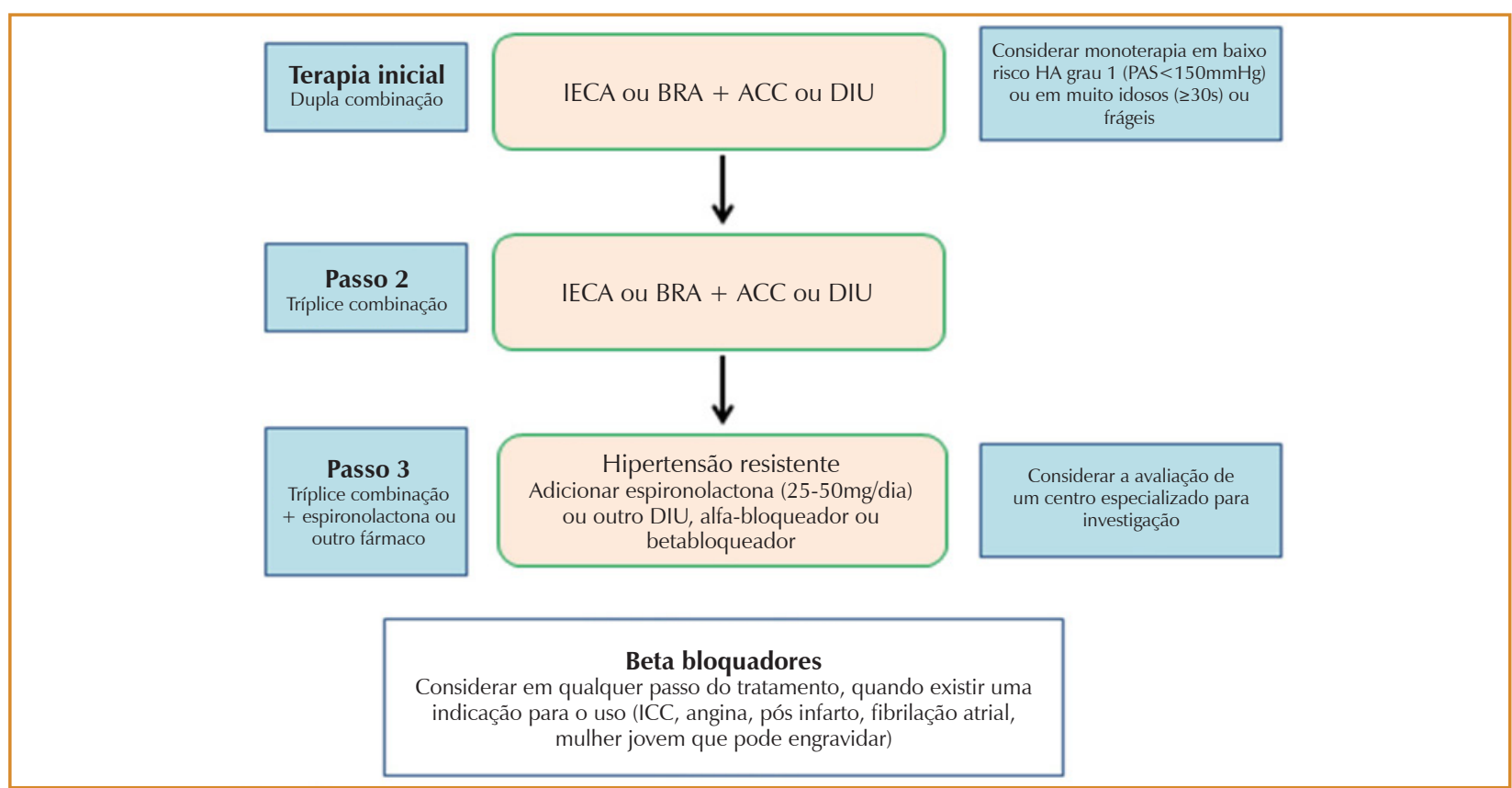

Figura 3. Fluxograma da estratégia de tratamento da hipertensão não complicada. Legenda: IECA (inibidor da enzima conversora da angiotensina); BRA (bloqueador do receptor da angiotensina); ACC (antagonista dos canais de cálcio). Modificado da referência 13. 
síndrome metabólica além da hipertensão arterial. Pacientes com diabetes foram excluídos do estudo.

Os pacientes foram designados aleatoriamente para tratamento oral diário ao longo de 24 semanas com doses iniciais de $10 \mathrm{mg}$ de amilorida, $25 \mathrm{mg}$ de hidroclorotiazida (HCTZ) ou $5 \mathrm{mg}$ de amilorida mais 12,5 mg de HCTZ. Todas as doses foram dobradas após 12 semanas. A aleatoriedade foi realizada por meio de computador e ambos os participantes e pesquisadores não conheciam os fármacos.

O desfecho primário do estudo foi a mudança da linha de base da concentração de glicose no plasma após 2 horas da ingesta de 75 g de glicose oral em 12 e 24 semanas e o desfecho secundário foi a mudança da linha de base da pressão residencial na $12^{\circ}$ e $24 \%$ semana.

Entre novembro de 2009 e dezembro de 2014 foram selecionados 663 pacientes, porém 222 foram excluídos da amostra. Os 441 pacientes restantes que respeitavam os critérios de inclusão foram alocados em grupos: 145 no grupo da amilorida, 146 no grupo da HCTZ e 150 no grupo com a combinação HCTZ -amilorida. O estudo tinha a intenção de tratar com 12 e 24 semanas.

A alteração média da linha de base da glicose plasmática após 2 horas da ingesta de $75 \mathrm{~g}$ de glicose foi de - 0,55 mmol/L (95\% IC, $-0,96$ a $-0,14, p=0,093)$ no grupo amilorida versus o grupo HCTZ quando as medidas foram na $12^{\circ}$ semana e na 24 a semana. Como a diferença foi significante, o estudo avaliou a média da linha de base da glicose plasmática após 2 horas da ingesta de $75 \mathrm{~g}$ de glicose no grupo que combinou amilorida e HCTZ versus grupo tiazídico. A associação apresentou uma alteração média de -0,42 mmol/L (95\% IC, -0.84 a-0.004] p=0.048).

A redução da pressão arterial sistólica residencial durante as 24 semanas não diferiu significantemente entre o grupo da amilorida e da HCTZ, mas queda da pressão arterial no grupo da combinação foi maior que o grupo HCTZ $(p=0,0068)$. A redução média da pressão arterial sistólica domiciliar em
24 semanas foi de $-12,9 \mathrm{mmHg}$ (IC 95\% - 14, , $\mathrm{a}-11,2)$ no grupo amilorida, $-12,2 \mathrm{mmHg}(-13,9$ a $-10,5)$ no grupo da HCTZ e -15,6 mmHg (-17,3 s -13,8) no grupo combinação. A queda da pressão arterial sistólica domiciliar foi, em média, 3,4 mmHg $(0,9$ a 5,8) maior no grupo combinação em comparação com o grupo HCTZ ao longo de 24 semanas. As mudanças na pressão arterial de consultório ao longo das 24 semanas não diferiram significativamente entre os grupos da amilorida e HCTZ. A combinação resultou em uma queda da pressão arterial em -20 mmHg ( -22,4 a 18,4), significativamente maior em comparação com o grupo da HCTZ.

O estudo PATHWAY-3 demonstrou que após 24 semanas, um diurético poupador de potássio reduz a pressão arterial tão eficaz quanto em altas doses de tiazídicos sem efeitos adversos na glicemia. Além disso, uma combinação com metade das doses convencionais de amilorida e HCTZ não se associou ao aumento da concentração de glicose em comparação com o tratamento com HCTZ sozinho, mas apresentou reduções significativamente maiores na pressão arterial em comparação com qualquer diurético, em doses alvo, administrados sozinhos. (Figura 4)

Finalmente, a combinação de amilorida com HCTZ previne a intolerância a glicose e melhora o controle pressórico em comparação com a terapia de base com qualquer um dos medicamentos.

Estes dois estudos, coordenados pelo professor Bryan Williams são fundamentais para compreender a complexa relação da HAR com os diversos fármacos anti-hipertensivos, expondo um conhecimento importante da fisiopatologia desta doença e sua correlação com a retenção salina. Além disso, a associação de um diurético que potencialmente pode provocar espoliação de potássio, com outro diurético poupador, pode ser útil e benéfico sem os efeitos adversos do primeiro. Os dois estudos, Pathway 2 e 3 , com objetivos próprios que convergem para um mesmo destino no controle do refratário, são de importância fundamental para se entender a complexa rede etiopatológica da hipertensão resistente.

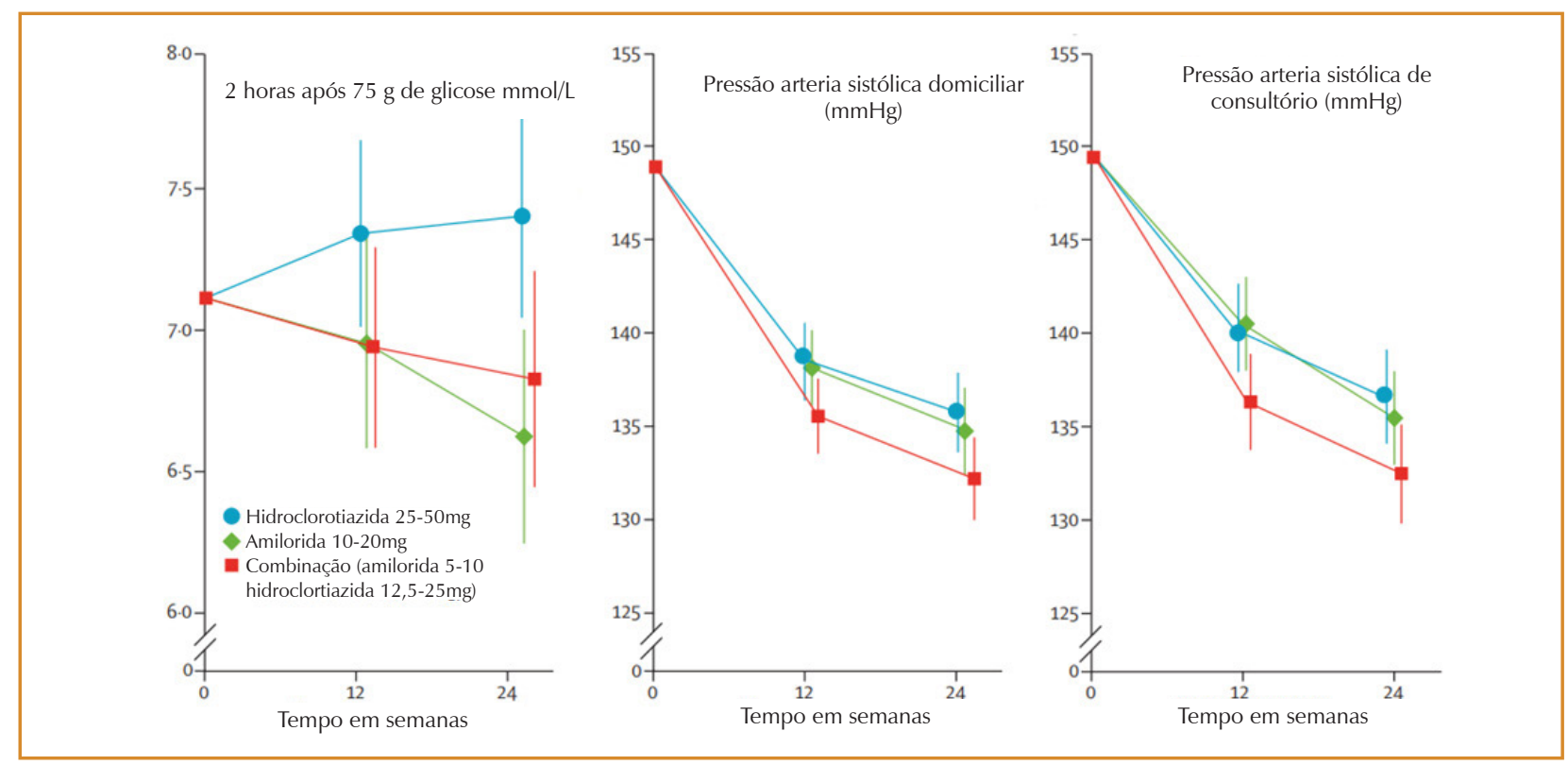

Figura 4. Mudança na concentração 2 h após 75 g de glicose, pressão arterial sistólica domiciliar e pressão arterial sistólica de consultório. Modificado da referência 31. 


\section{REFERÊNCIAS}

1. Carey RM, Calhoun DA, Bakris GLO, et al. Resistant hypertension: detection, evaluation and management. A scientific statement from the American Heart Association. Hypertension. 2018;72:e53-e90. doi: 10.1161/ HYP.0000000000000084.

2. Pimenta E, Gaddam KK, Pratt-Ubunama MN, S et al. Relation of dietary salt and aldosterone to urinary protein excretion in subjects with resistant hypertension. Hypertension. 2008;51:339-44. doi: 10.1161/ HYPERTENSIONAHA.107.100701

3. Drager LF, Lopes HF, Maki-Nunes C, et al. The impact of obstructive sleep apnea on metabolic and inflammatory markers in consecutive patients with metabolic syndrome. PLoS One. 2010;5:e12065. doi: 10.1371/journal. pone.0012065.

4. Pedrosa RP, Drager LF, Gonzaga CC, Sousa MG, de Paula LK, Amaro AC et al. Obstructive sleep apnea: the most common secondary cause of hypertension associated with resistant hypertension. Hypertension. 2011;58:811-7. doi: 10.1161/HYPERTENSIONAHA.111.179788.

5. Howard BV, Rodriguez BL, BennettPH, etal. Prevention conference VI: diabetes and cardiovascular disease: Writing Group I: epidemiology. Circulation. 2002;105:e132-e137. doi: 10.1161/01.cir.0000013953.41667.09.

6. Calhoun DA, Jones D, Textor S, et al. Resistant hypertension: diagnosis, evaluation, and treatment. A scientific statement from the American Heart Association Professional Education Committee of the Council for High Blood Pressure Research. Hypertension. 2008 Jun;51(6):1403-19. doi: 10.1161/ HYPERTENSIONAHA.108.189141. .

7. Whelton PK, Carey RM, Aronow WS, et al. 2017 ACC/AHA/AAPA/ABC/ ACPM/AGS/APhA/ASH/ASPC/NMA/PCNA guideline for the prevention, detection, evaluation, and management of high blood pressure in adults: a report of the American College of Cardiology/American Heart Association Task Force on Clinical Practice Guidelines. Hypertension. 2018;71:e13e115. doi: 10.1161/HYP.0000000000000065.

8. Persell SD. Prevalence of resistant hypertension in the UnitedStates, 2003-2008. Hypertension. 2011;57:1076-80. doi: 10.1161/ HYPERTENSIONAHA.111.170308.

9. Lotufo PA, Pereira AC, Vasconcellos PS, Santos IS, Mill JG, Bensenor IM. Resistant hypertension: risk factors, subclinical atherosclerosis, and comorbidities among adults-the Brazilian Longitudinal Study of Adult Health (ELSA-Brasil). J Clin Hypertens. 2015;17:74-80. doi: 10.1111/jch.12433.

10. Daugherty SL, Powers JD, Magid DJ, et al. Incidence and prognosis of resistant hypertension in hypertensive patients. Circulation. 2012;125:1635-42. doi: 10.1161/CIRCULATIONAHA.111.068064.

11. Salles GF, Cardoso CR, Muxfeldt ES. Prognostic influence of office and ambulatory blood pressures in resistant hypertension. Arch Intern Med. 2008;168:2340-6. doi: 10.1001/archinte.168.21.2340.

12. Chobanian AV, Bakris GL, Black HR, et al. Joint National Committee on Prevention, Detection, Evaluation, and Treatment of High Blood Pressure. National Heart, Lung, and Blood Institute; National High Blood Pressure Education Program Coordinating Committee. The Seventh Report of the Joint National Committee on Prevention, Detection, Evaluation, and Treatment of High Blood Pressure. The JNC 7 Report. Hypertension. 2003; 42: 1206-52. doi: 10.1161/01.HYP.0000107251.49515.c2.

13. Mancia G, Laurent S, Agabiti-Rosei E, et al. Reappraisal of European Guidelines on hypertension management: a European Society of Hypertension Task Force document. J Hypertens. 2009; 27:2121-58. doi: 10.1097/HJH.0b013e328333146d.

14. Irvin MR, Shimbo D, Mann DM, et al. Prevalence and correlates of low medication adherence in apparent treatmentresistant hypertension. J Clin Hypertens 2012; 14: 694-700. doi: 10.1111/j.1751-7176.2012.00690.x

15. Muxfeldt ES, Salles GF. How to use ambulatory blood pressure monitoring in resistant hypertension. Hypertens Res. 2013; 36:385-9. doi: 10.1038/ hr.2013.17. Epub 2013 Mar 21.
16. De la Sierra A, Segura J, Banegas JR, et al. Clinical features of 8295 patients with resistant hypertension classified on the basis of ambulatory blood pressure monitoring. Hypertension. 2011; 57: 898-902. doi: 10.1161/ HYPERTENSIONAHA.110.168948.

17. Sim JJ, Bhandari SK, Shi J, et al. Comparative risk of renal, cardiovascular, and mortality outcomes in controlled, uncontrolled resistant, and nonresistant hypertension. Kidney Int. 2015; 88(3):622-32. doi: 10.1038/ki.2015.142.

18. de Souza F, Muxfeldt ES, Salles GF. Prognostic factors in resistant hypertension: implications for cardiovascular risk stratification and therapeutic management. Expert Rev Cardiovasc Ther. 2012;10:735-45. doi: 10.1586/erc.12.58

19. Calhoun DA. Hyperaldosteronism as a common cause of resistan hypertension. Annu Rev Med 2013;64:233-47. doi: 10.1146/annurevmed-042711-135929.

20. Clark D, Ahmed MI, Calhoun DA. Resistant hypertension and aldosterone: an update. Can J Cardiol 2012;28:318-25. doi: 10.1016/j.cjca.2012.03.010.

21. Pimenta E, Gaddam KK, Oparil S, et al. Effects of dietary sodium reduction on blood pressure in subjects with resistant hypertension: results from a randomized trial. Hypertension. 2009;54(3):475-81. doi: 10.1161/ HYPERTENSIONAHA.109.131235.

22. Parati G, Lombardi C, Hedner J, et al. Position paper on the management of patients with obstructive sleep apnea and hypertension: joint recommendations by the European Society of Hypertension, by the European Respiratory Society and by the members of European COST (COoperation in Scientific and Technological research) ACTION B26 on obstructive sleep apnea. J Hypertens. 2012;30: 633-46.

23. Barbe' F, Duran-Cantolla J, Capote F, et al. Long term effect of continuous positive airway pressure in hypertensive patients with sleep apnea. Am J Respir Crit Care Med. 2010;181:718-26. doi: 10.1164/rccm.2009010050OC.

24. De la Sierra A, Banegas JR, Oliveras A, Gorostidi M, Segura J, de la Cruz JJ et al. Clinical differences between resistant hypertensives and patients treated and controlled with three or less drugs. J Hypertens. 2012;30:1211-16. doi: 10.1097/HJH.0b013e328353634e.

25. Williams B, MacDonald TM, Morant S, et al. Spironolactone versus placebo, bisoprolol, and doxazosin to determine the optimal treatment for drugresistant hypertension (PATHWAY-2): a randomised, double-blind, crossover trial. Lancet. 2015;386:2059-68. doi: 10.1016/S0140-6736(15)00257-3.

26. Guo H, Xiao Q. Clinical efficacy of spironolactone for resistant hypertension: a meta analysis from randomized controlled clinical trials. Int J Clin Exp Med. 2015;8:7270-8.

27. Zillich AJ, Garg J, Basu S, Bakris GL, Carter BL. Thiazide diuretics, potassium and the development of diabetes: a quantitative review. Hypertension. 2006;48:219-24. doi: 10.1161/01.HYP.0000231552.10054.aa.

28. Cutler JA. Thiazide-Associated glucose abnormalities: prognosis, etiology, and prevention: is potassium balance the key? Hypertension. 2006;48:198200. doi: 10.1161/01.HYP.0000231339.51310.b3.

29. Duarte JD, Cooper-DeHoff RM. Mechanisms for blood pressure lowering and metabolic eff ects of thiazide and thiazide-like diuretics. Expert Rev Cardiovasc Ther. 2010;8:793-802. doi: 10.1586/erc.10.27.

30. Mackenzie IS, Brown MJ. Molecular and clinical investigations in patients with low- renin hypertension. Clin Exp Nephrol. 2009;13:1-8. doi: 10.1007/s10157-008-0071-4.

31. Brown MJ, Williams B, Morant SV, Webb DJ, Caulfield MJ, Cruickshank JK et al. Effect of amiloride, or amiloride plus hydrochlorothiazide, versus hydrochlorothiazide on glucose tolerance and blood pressure (PATHWAY-3): a parallel-group, double-blind randomized phase 4 trial. Lancet Diabetes Endocrinol. 2016:4:136-47. doi: 10.1016/S2213-8587(15)00377-0. 